Journal Of Al-Azhar University Engineering Sector

Vol. 14, No. 51, April, 2019, 653-669

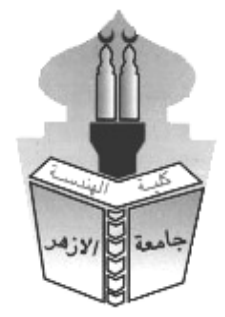

\title{
DESIGN AND BUILDING AN AUTOMATED MEASUREMENT AND CONTROL SYSTEM
}

\author{
Hend Mohamed Elsaied Soliman Mosbah and Ahmed Mohamed N. Elmahdy \\ Computer and System Dep., Faculty of Engineering, Al-Azhar University
}

\begin{abstract}
Measurement and control systems (MCS) are the most important systems in all levels and fields, whether in our daily life such as controlling the temperature of a house and its luminance and ..., or as the control of the electric elevator, a car protection system or a company protection system. As well as in the field of robotics and industry, where there is no factory of any kind does not contain a system of measurement and control. And there are many applications for this systems in many technological fields, such as communications, transport, defense and many other fields. Therefore, there is an urgent need to know how to design and build systems, especially automated measurement systems and control flexibly under the circumstances and challenges that the designer meet them from the surrounding environment him or surrounding the system itself.

Here, some solutions have been found to design and build the automated measurement and control systems flexibly, which requires to maintain the effort in terms of design, building or using, as required by the system itself without compromising the efficiency of its work (reaching to optimization). And thus achieve flexibility in use the system, and tune it with what is required of it.

Keywords: Design and building system, automated measurement and control system, Sensor, Communication Protocols

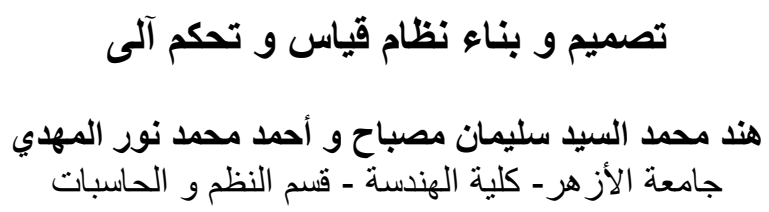




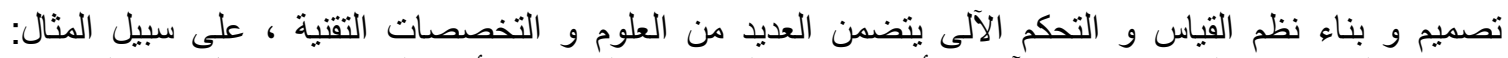

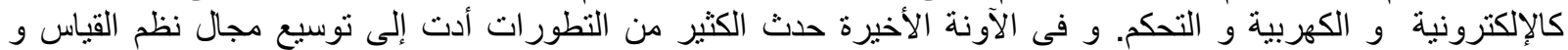

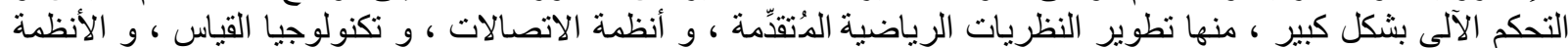
الرقمية ، و كذللك التفاعل بين الإنسان و الآلة.

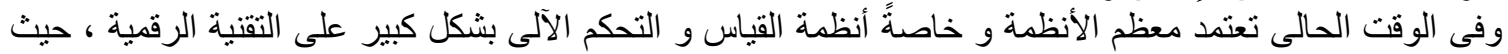

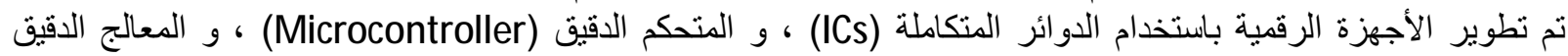

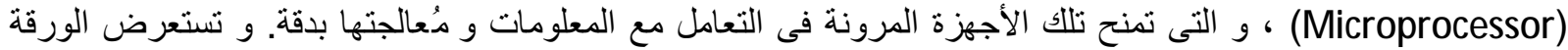
البحثية طرق تصميم و بناء تلك النظم.

Challenges " r - طرق تنفيذ تصميم و بناء نظم القياس و التئ التحمم الآلى:

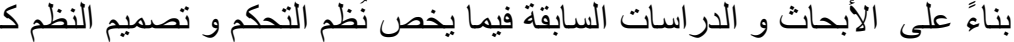
Digital control system of the recuperation device" ، "(1)"and Types of Home Automation Systems On System " ، (3)" Devices for Automatic Measurement Contemporary Methods and"، (2)" ، ${ }^{(6)}$ "Resilient Control System Execution Agent"، (5)"Resilient Control Systems" “"(4)"Design Home Appliance " ، ${ }^{(7) " E x p e r i m e n t a l ~ v a l i d a t i o n ~ o f ~ a ~ r e s i l i e n t ~ m o n i t o r i n g ~ a n d ~ c o n t r o l ~ s y s t e m " ~}$ Management System for Monitoring Digitized Devices Using Cloud Computing Technology in

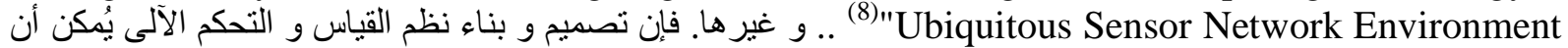
تتم بإحدى الطرق الثلاثة الآتية أو جميعُها ، كالآتى :

الطريقة الأولى: و يتم فيها التحكم فى الأجهزة المتواجدة فى مكان واحد و فى نفس الحيز ، حيث يكون ذلك غالباً باستخدام المتحكم الدقيق (Microcontroller)

الطريقة الثانية: كيفية التحكم فى الأجهزة المتواجدة فى مسافات أبعد فى نفس المكان أو الموقع مثل: المصانع و المعامل ، و و

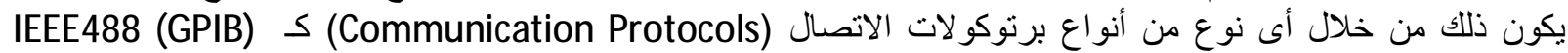

. protocol

الطريقة الثالثة: التحكم فى الأجهزة عن بعد ، مثل: المواقع الحربية أو الأماكن التى يوجد بها خطورة أثناء التواجد بها بها

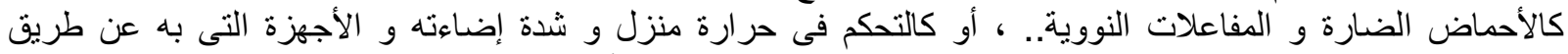
الموبايل،و غير ها من التطبيقات التى لاحصر لها. و يكون ذلك من خلال أى نوع من الـ

بـ الأسلوب الأمثل لتصميم نظام القياس والتحكم الآلى:

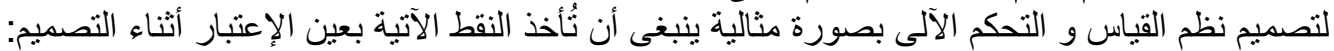

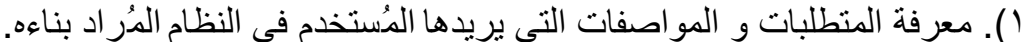

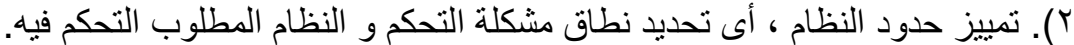

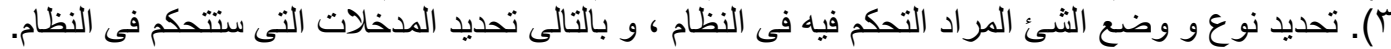

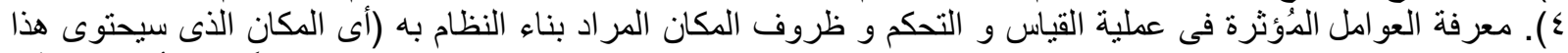

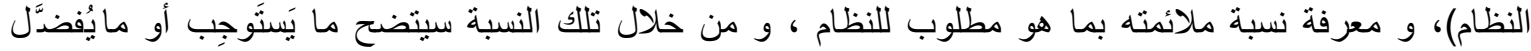

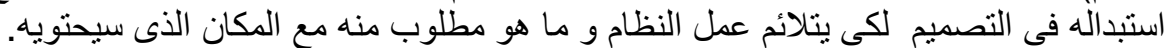

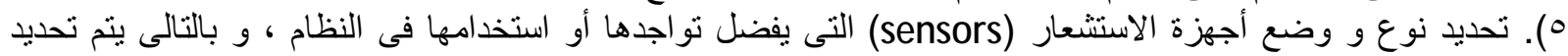

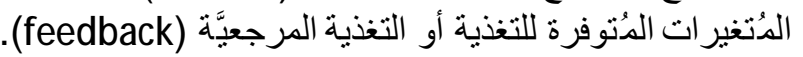

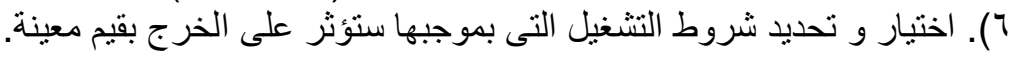




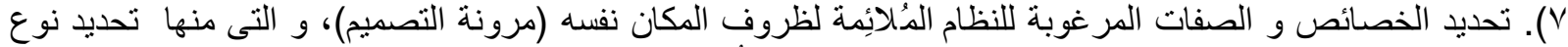

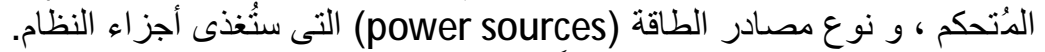

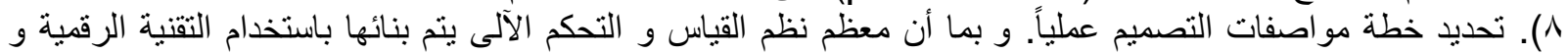

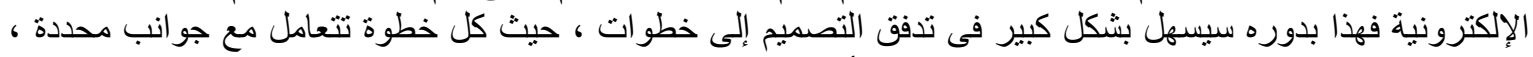

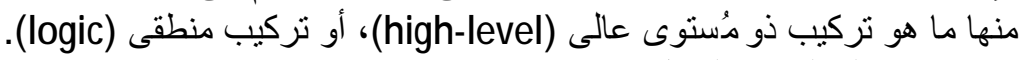
9). تصميم و هيكلة النظام بالكامل. • ( ). تطبيق مبادئ الصحة و السلامة أثناء التصميح.

ع ـ طرق تنفيذ تصميم و بناء نظم القياس و التحكم الآلى عملياً:

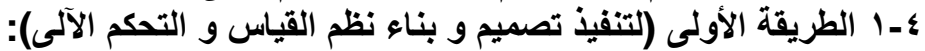

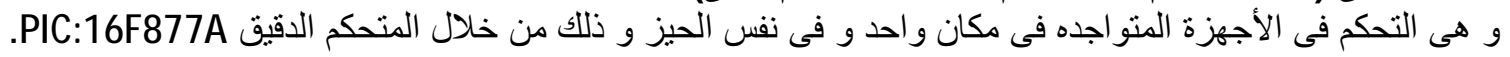
و معظم تطبيقها هذه الطريقة تكون في المعامل و المثاريع الصغان والصغيرة.

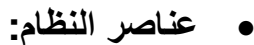

7-segment Displays, LCD 2×16 Display, LM 35, water Heater, thyristor , Keypad 4×4 with PIC:16F877A

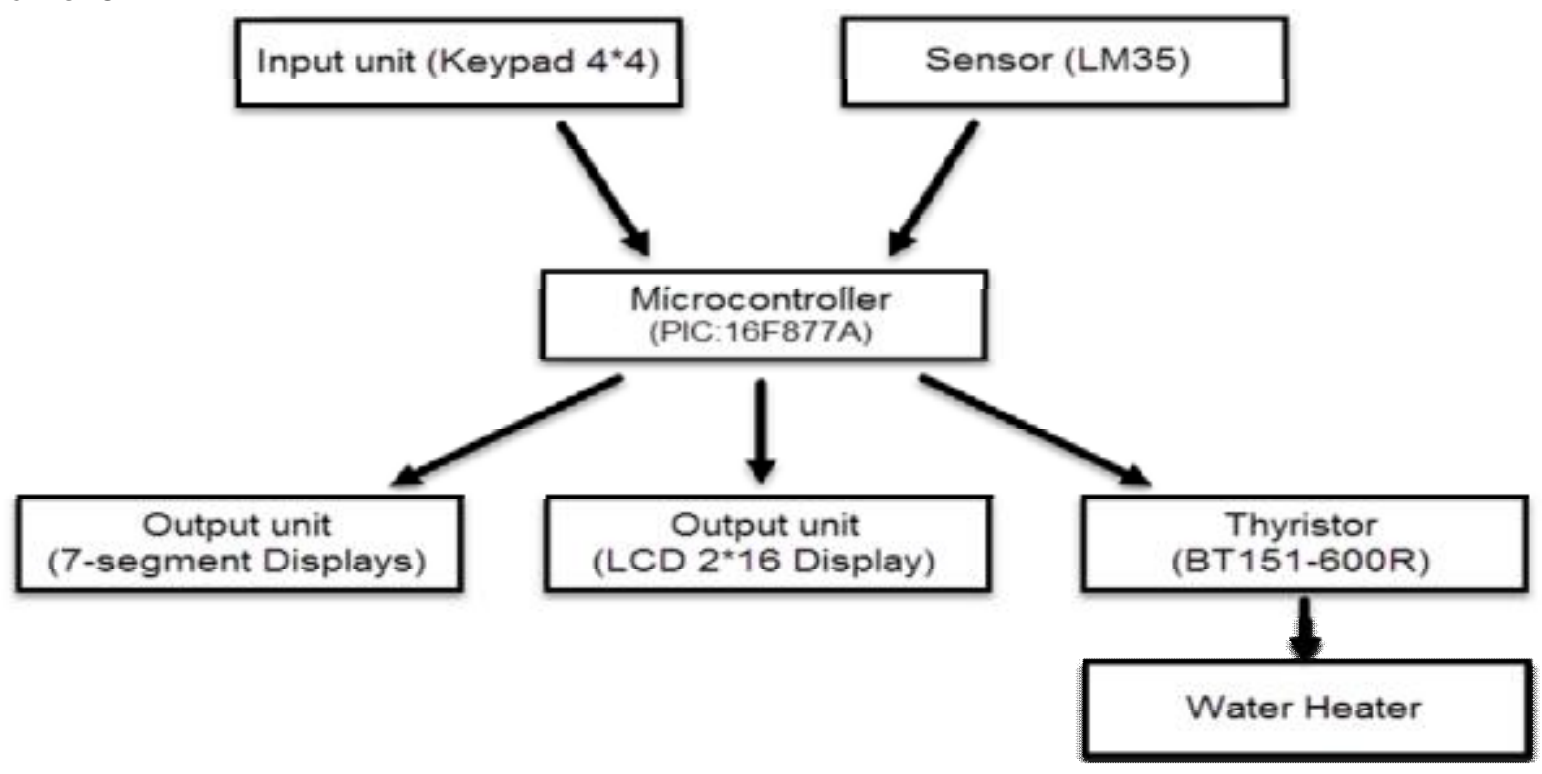

الشكل (1): الشكل العام للنظام

وظيفة هذا النظام : و ت و

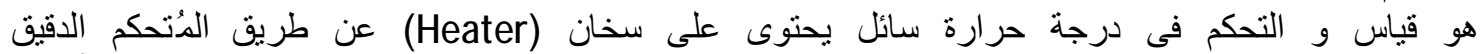

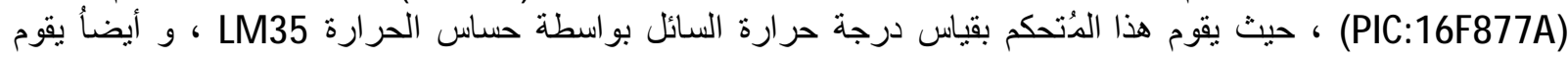

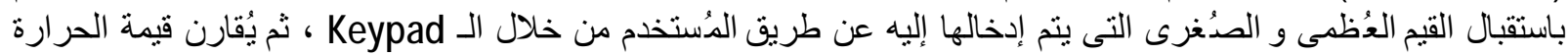

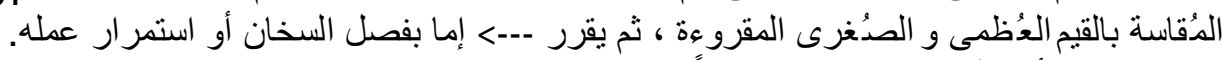

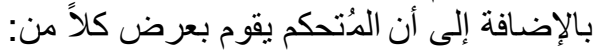

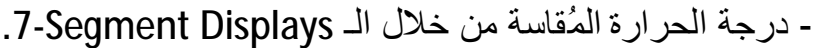

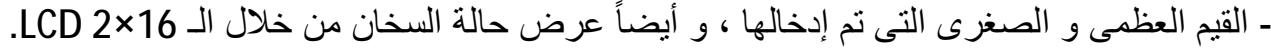

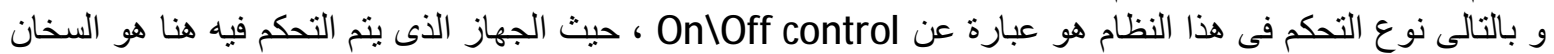

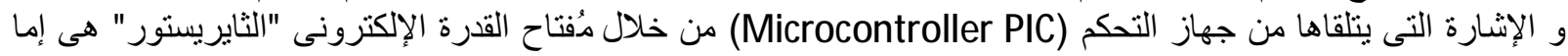

On=5v 
تصميم و بناء نظام قياس و تحكم آلى

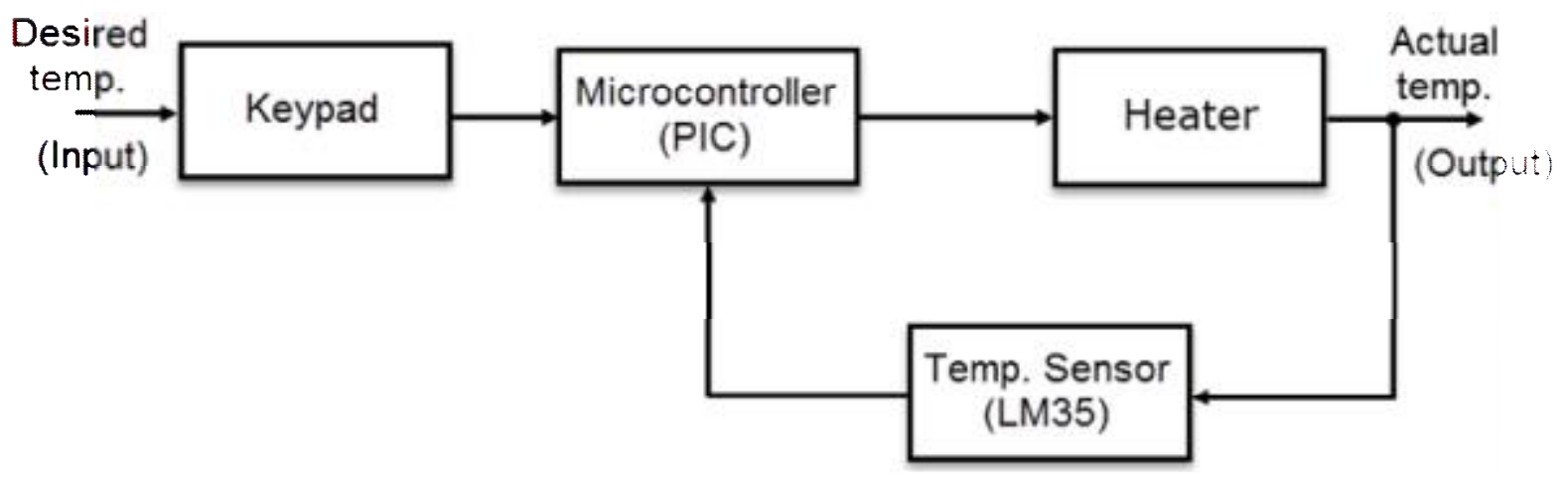

الثكل (ץ): الـ Block diagram الخاص بالنظام الأول

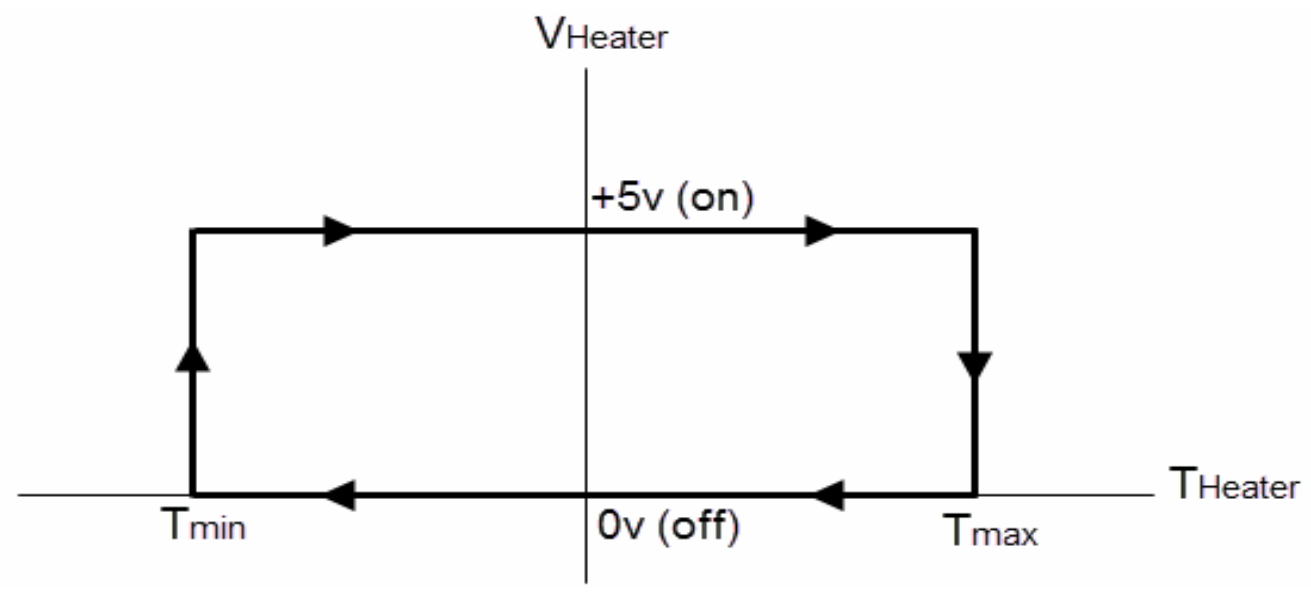

الثكل (ץ): الرسم البيانى لتوضيح العلاقة بين درجة الحرارة و خرج المُتحكم

حيث، Veater : هو قيمة خرج المتحكم الدقيق و الذى ينم توصبله بالسخان عن طريق الثايريستور. 


\section{"Temperature Control"}

\section{Current temp.}
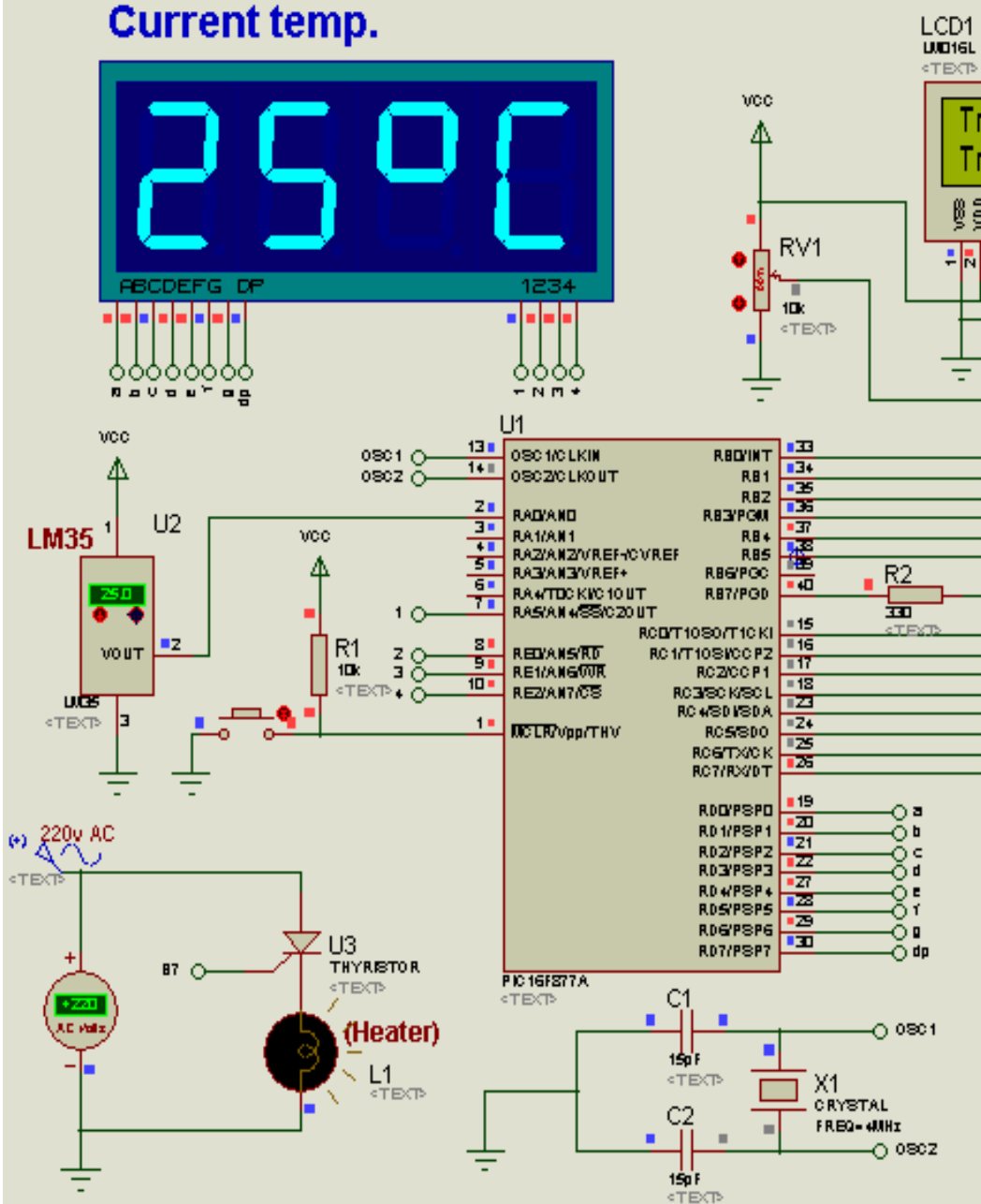

"Proteus" الثكل (؛): تصميم و تنفيذ دائرة النظام الإكترونية على برنامج المحاكاة للاوائر الإكترونية

ملاحظة:

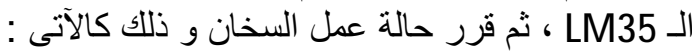
- If (Temp $>=$ Tmax) ---> Heater $=0$ (turn off)

- If (Temp <=Tmin) ---> Heater $=1$ (turn on)

\& ؟ r الطريقة الثانية(لتنفيذ تصميم و بناء نظم القياس و التحكم الآلى):

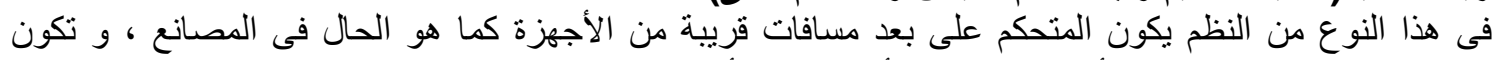

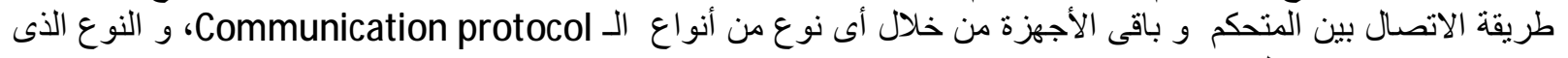

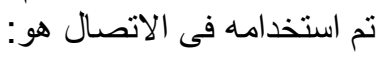
. IEEE488 bus communication (GPIB)

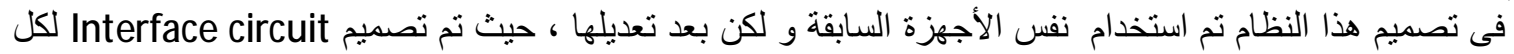

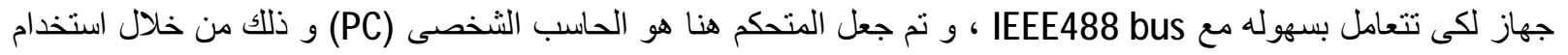
برنامج الـ LabVIEW 


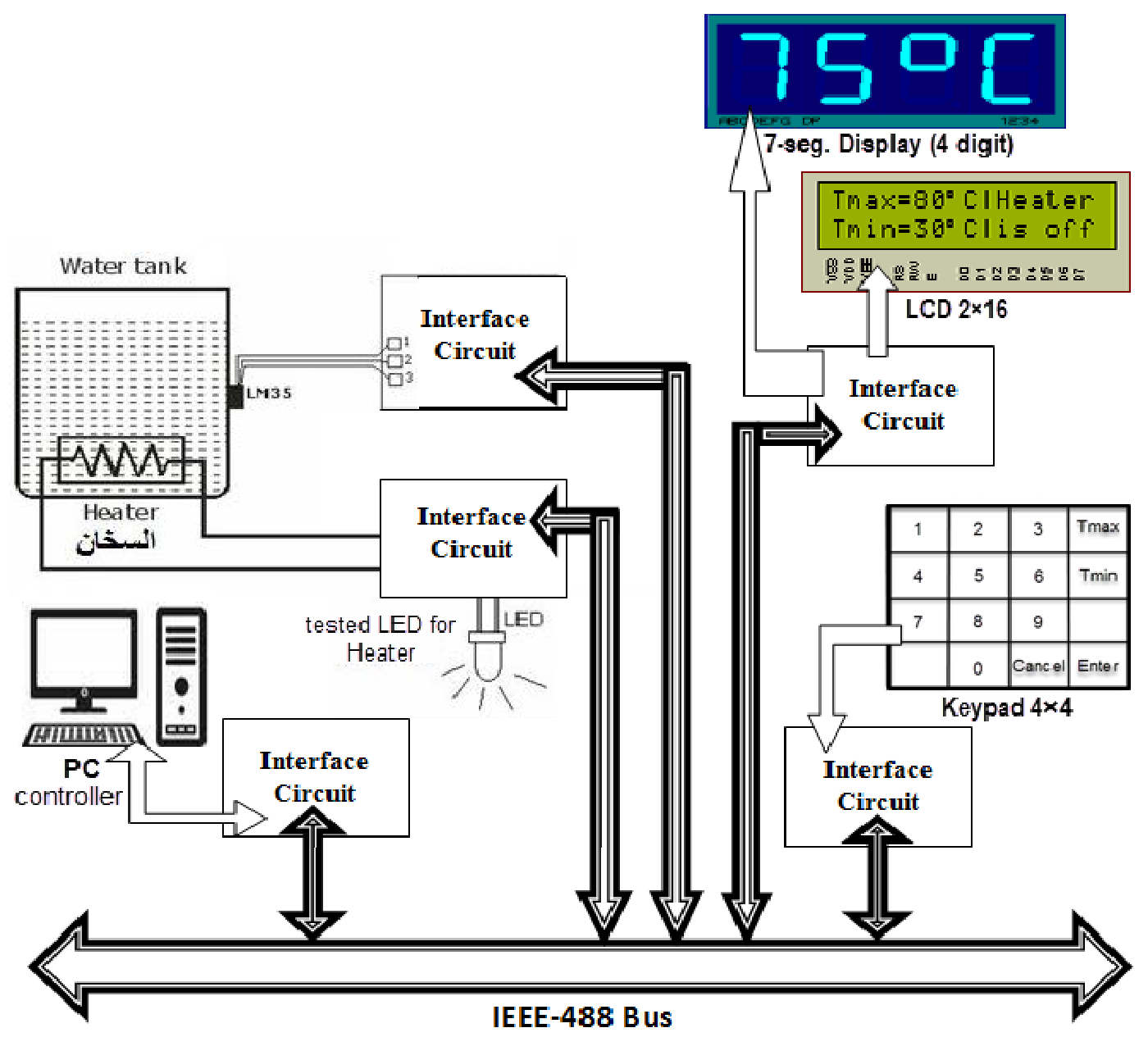

الشكل (•): الشكل العام للنظام

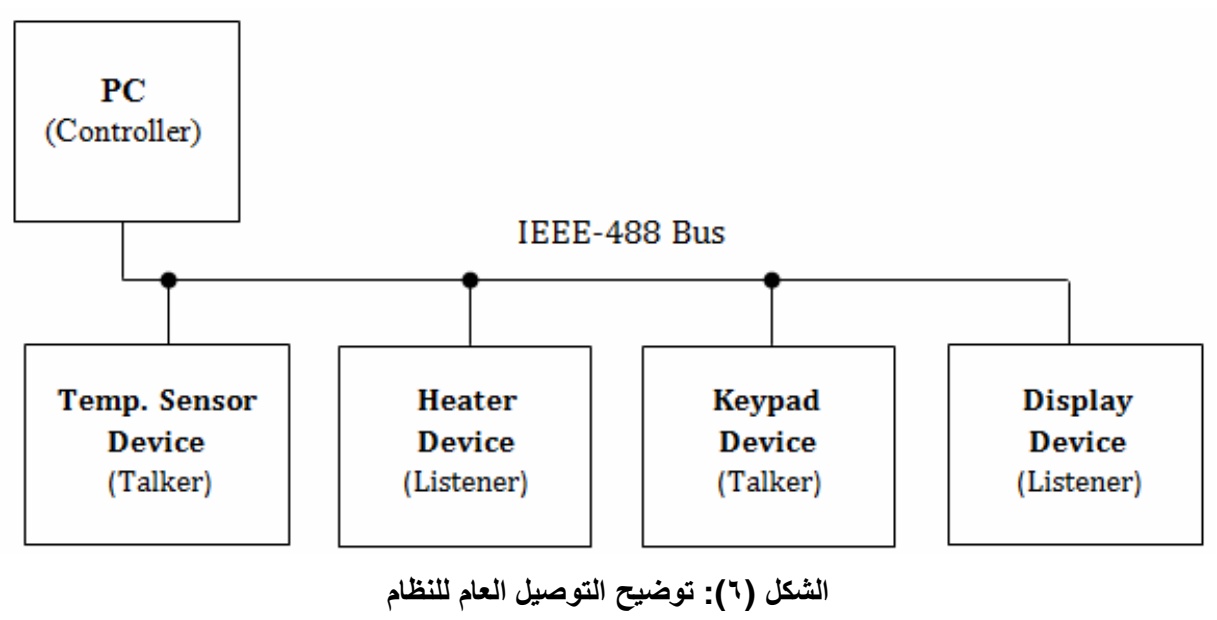


توضيح وظائفة خطوط مسار IEEE488 بشكل عام (9):

تستخدم فى نقل البيانات بين أجهزة و المُتحكم ، و أيضاً فى نقل الأوامر من المُتحكم إلى الأجهزة
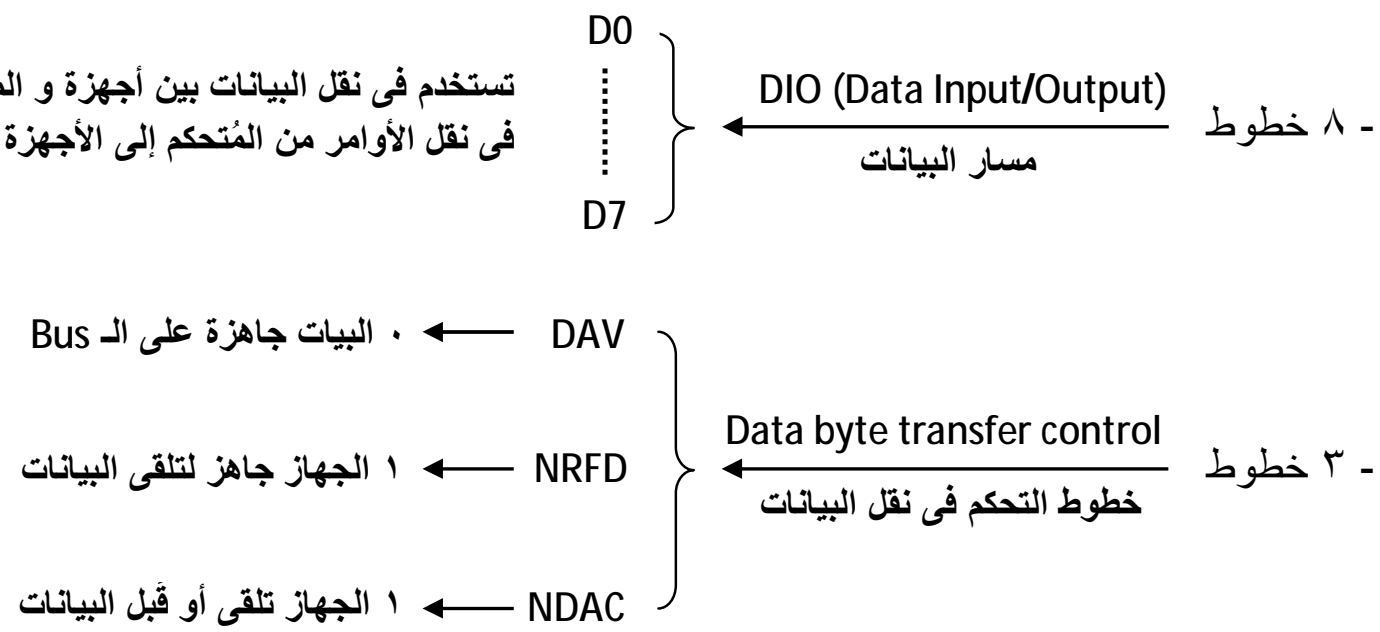

Data byte transfer control ـ ب خطوط خطوط التحكم فى نقل البيانات

(

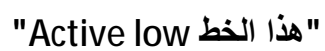

حالة نقل الأو امر و الغناوين في مسار

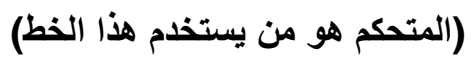

1 ـ إعادة ربط الـ IEE-488 IFC الحالة السابقة التعريف، كل الأجهزة تكون غير مُعنُوَنة

(المتحكم فقط هو من يستخدم هذا الخط ) الخرن

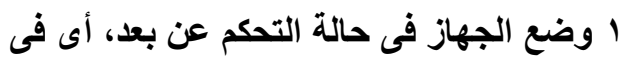
REN

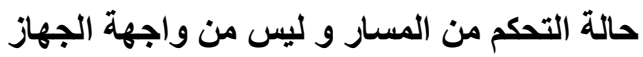

(المتحكم فقط هو من يستخدم هذا الخط )

ــ ـ SRQ

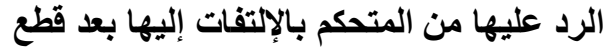

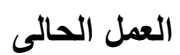

(تصدر من الجهاز الذى يطلب الخذمة )

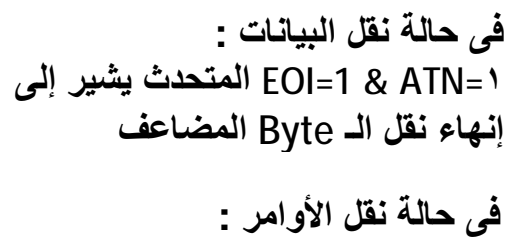

ATN

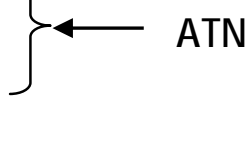

(1)

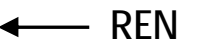

EOI=1 \& ATN=.

$$
\text { الأى تم انتخابه }
$$

(المتحكم هو من يستخدم هذا الخط) 


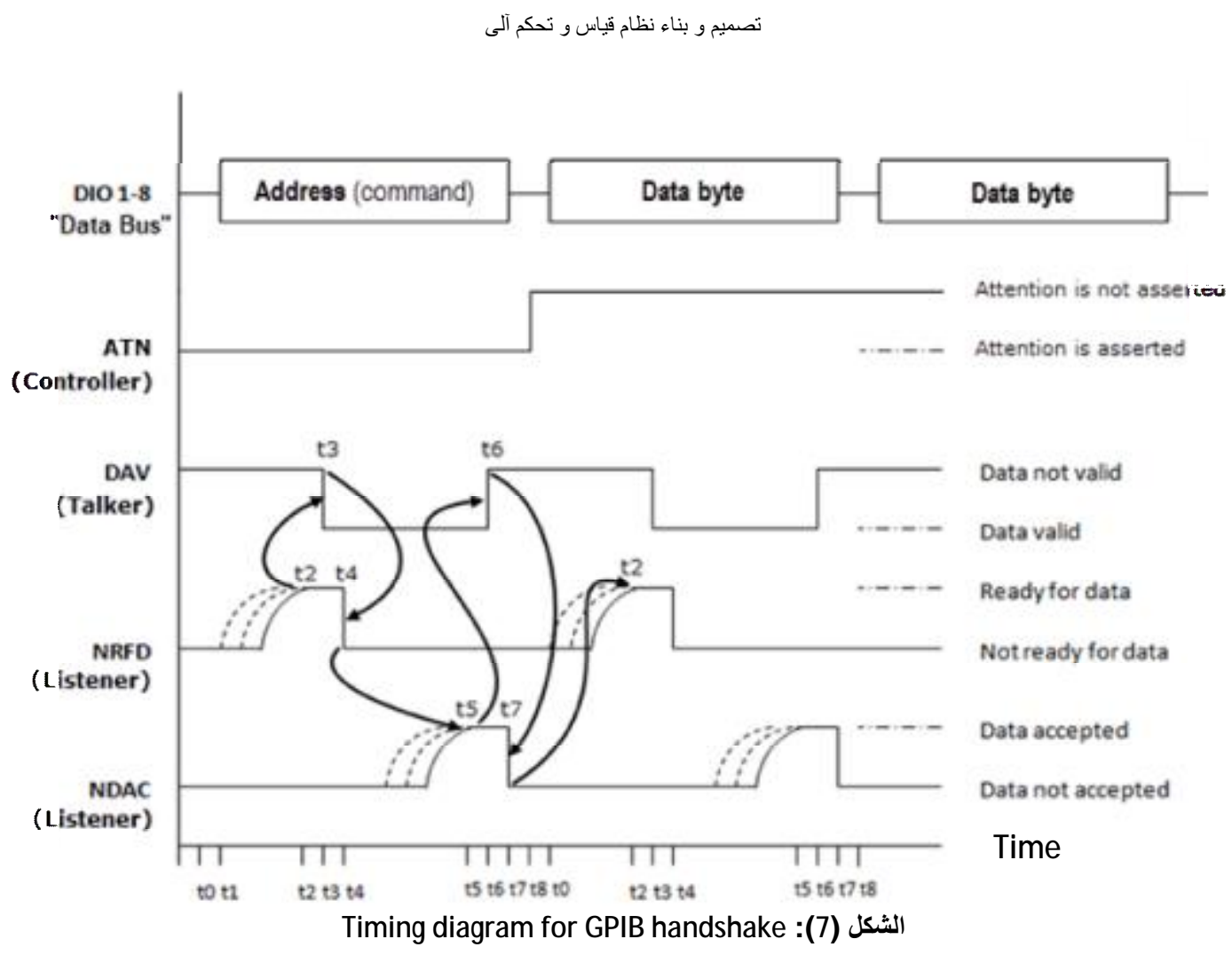

وظيفة هذا النظام (الطريقة الثانية):

وظيفة هذا التطبيق هى نفس وظيفة وظيفة نظام الطريقة الأولى باختلاف أن من سيُستخدَم هنا لغرض التحكم هو الحاسب

الثخصى PC و ليس المتحكم الدقيق PIC، و طريقة نقل البيانات تتم من خلال Bus communication (GPIB)

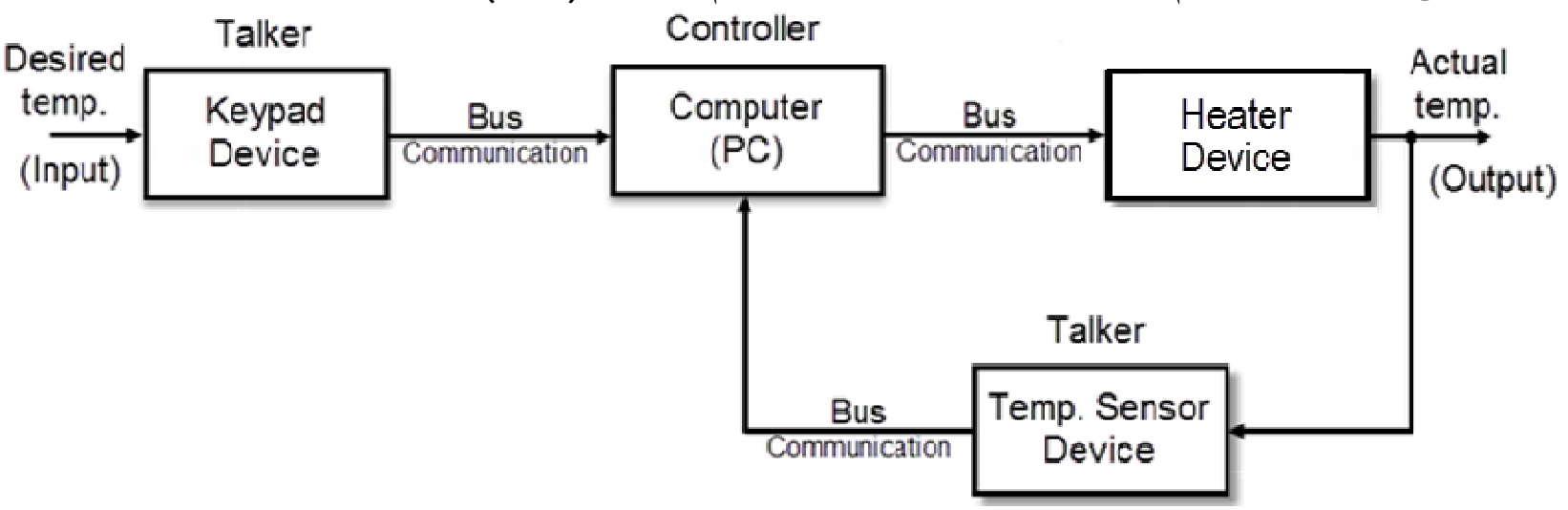

الثكل (8): الـ Block diagram الخاص بالنظام الثانى

: جهاز التحكم (PC controller)

جهاز التحكم ينقسم إلى قسمين : Software application \& USB-GPIB Interface circuit سنتطرق أو لاً إلى الـ USB-GPIB interface circuit و هي عبارة عن Interface circuit حلقة الوصل بين الـ PC و

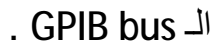

\section{: USB-GPIB interface circuit}

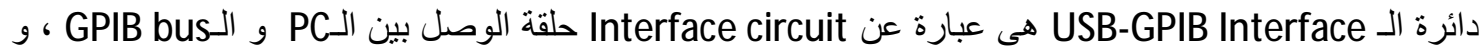

$$
\text { الدائرة التى تم استخدامها و تنفيذها هى كما بالثكل (9 ـ أ أ ، ب ، ج ج). }
$$




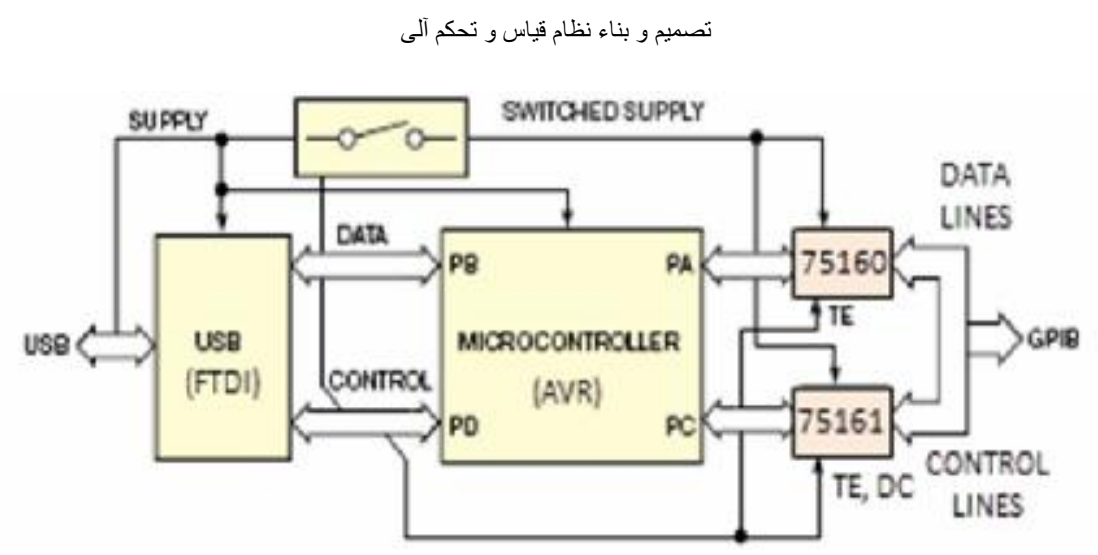

الثكل (9- أ): USB-GPIB Interface circuit من مرجع (10)

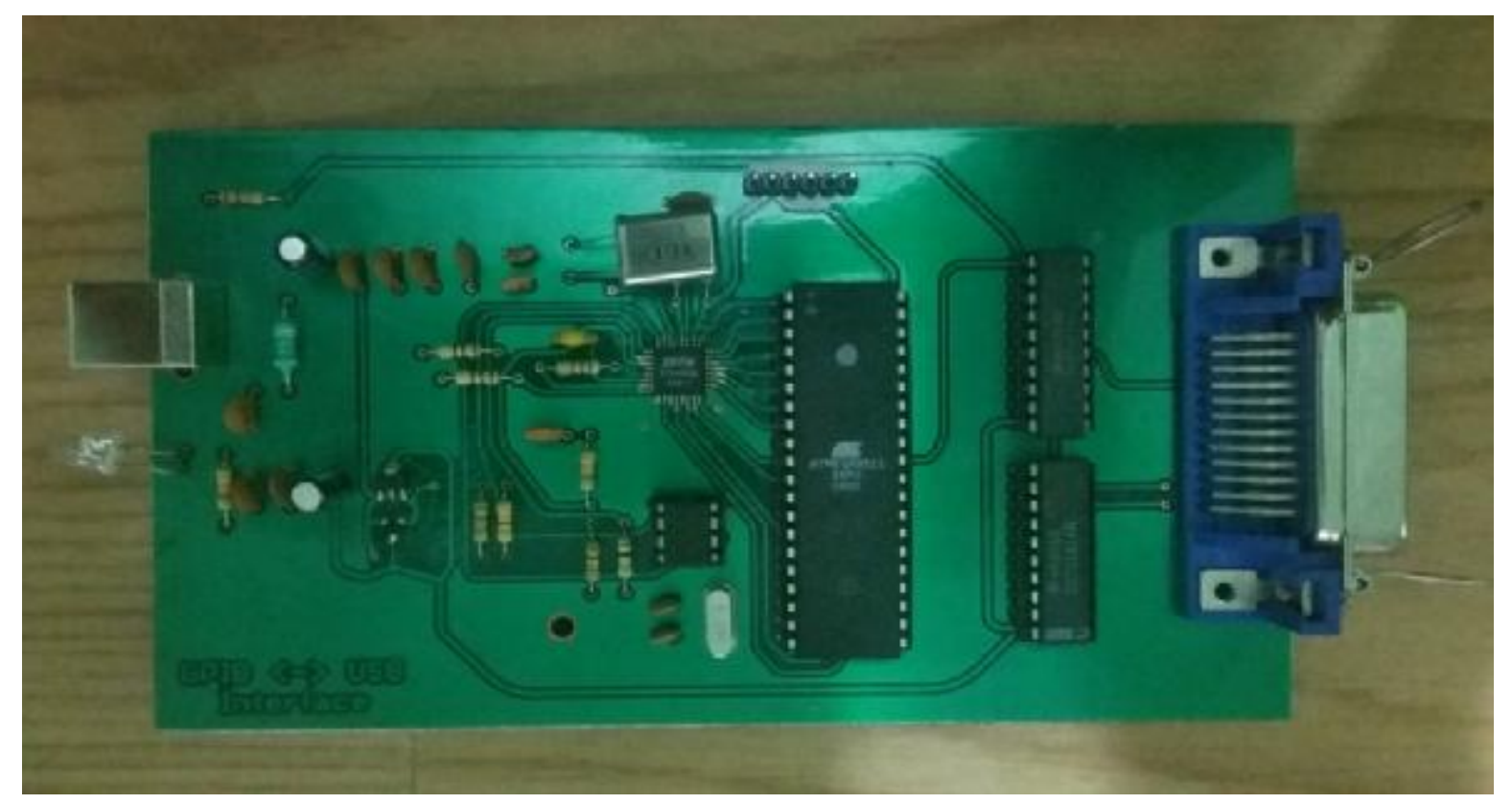

الشكل (9 - ج): تنفيذ دائرة الـ USB-GPIB Interface 


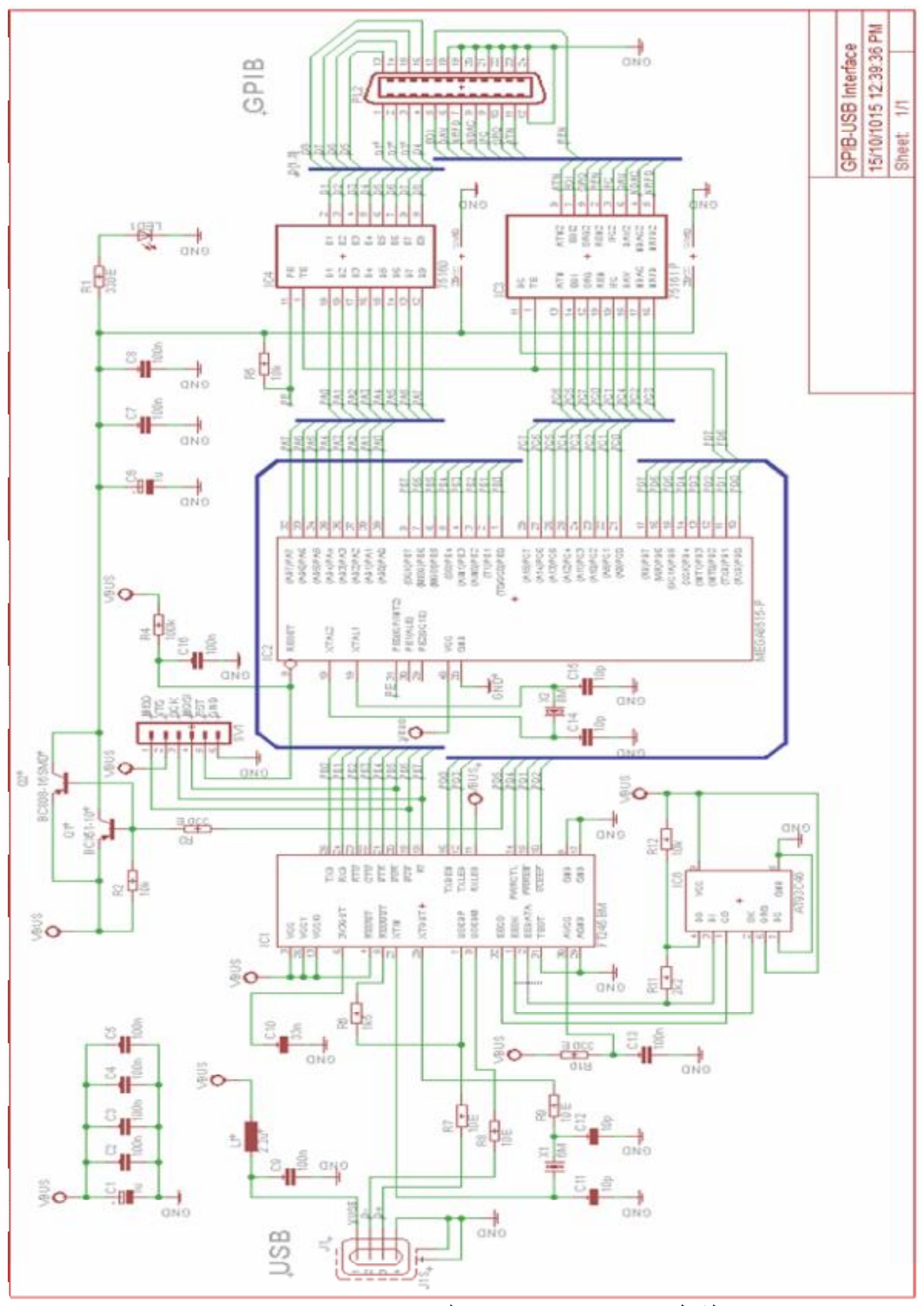

الثكل (9 - ب): تصميم دائرة الـ USB-GPIB Interface 
:Software Application -

الـ Software application هو التطبيق الذى تم تصميمه و بناءه لغرض الدُر اقبة و التحكم فى الأجهزة الدُتصلة بالمسار . (IEEE 488) GPIB

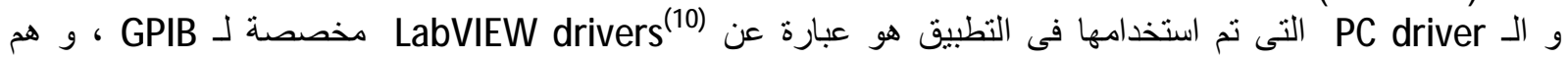

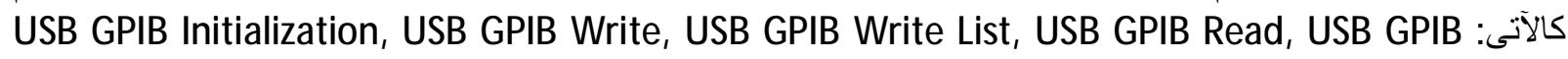
Clear, USB GPIB Read STB, USB GPIB Request Wait, USB GPIB Wait SRQ, USB GPIB Set timeout, USB GPIB Trigger, USB GPIB Trigger List and USB GPIB Close.

التطبيق الذى تم تصميمه و بناءه من خلال برنامج الـ LabVIEW و بمساعدة تلك الـ VIs ، هو كالآتى :

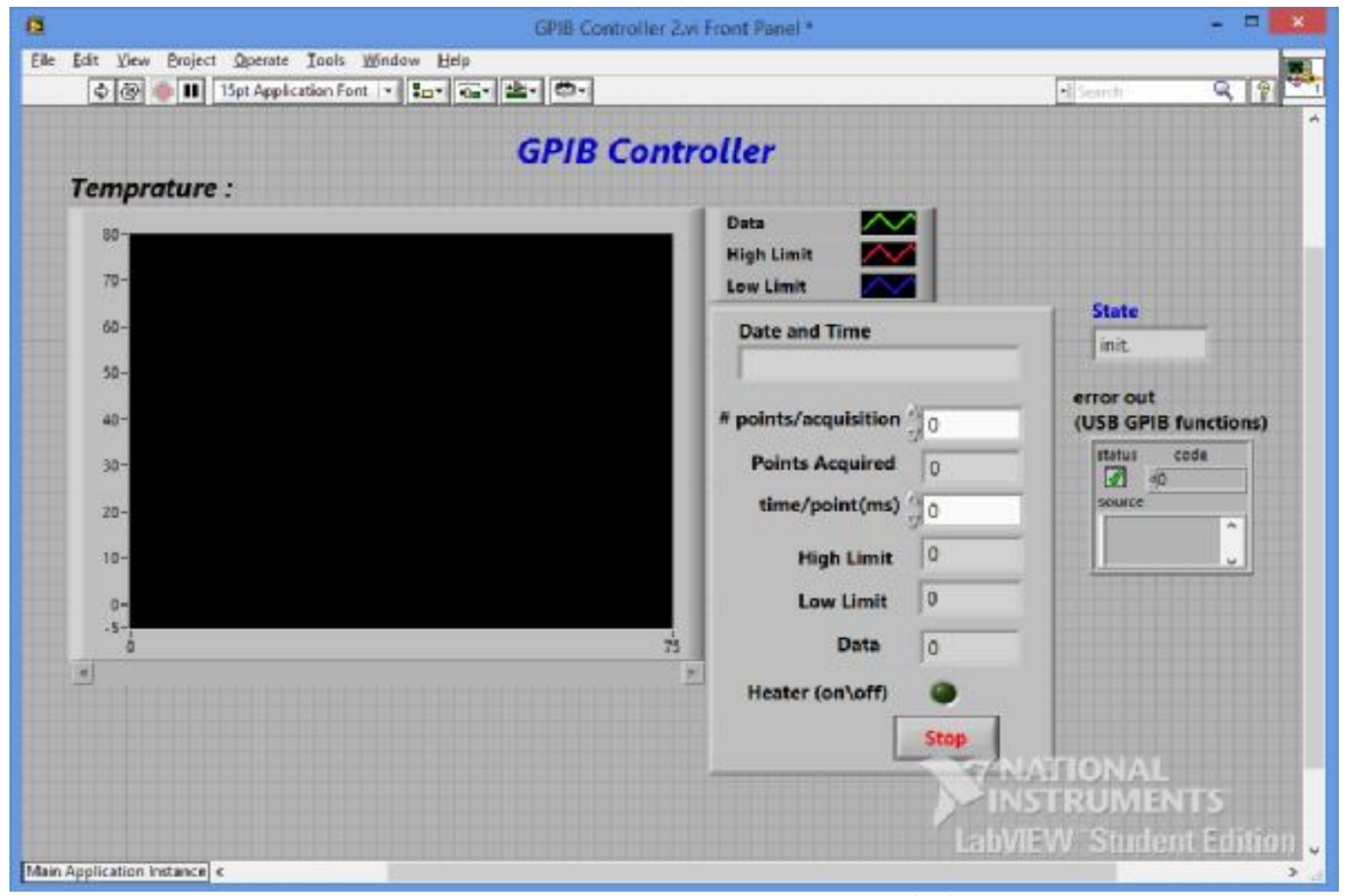

Front panel of GPIB control device application : (1 الشكل (1)

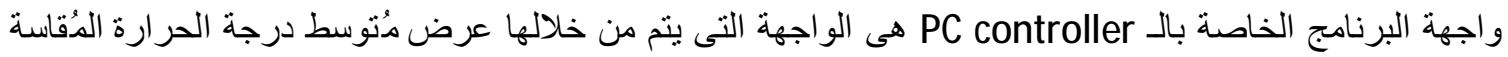

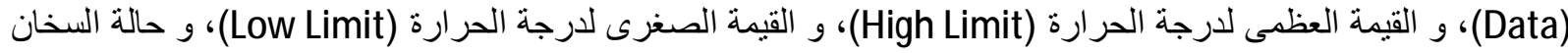
(Heater (on\off))

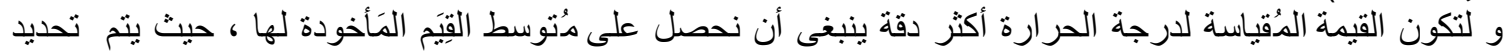

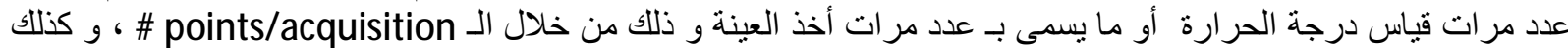

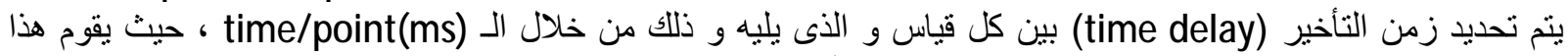

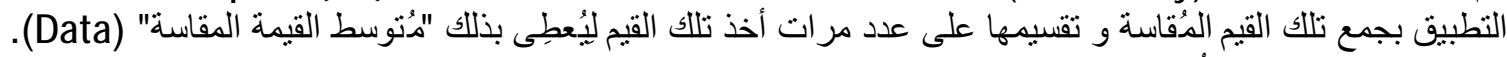

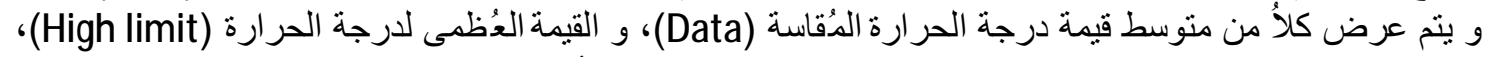

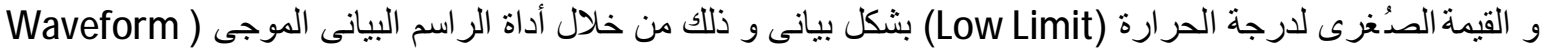
."Temprature" (charts 


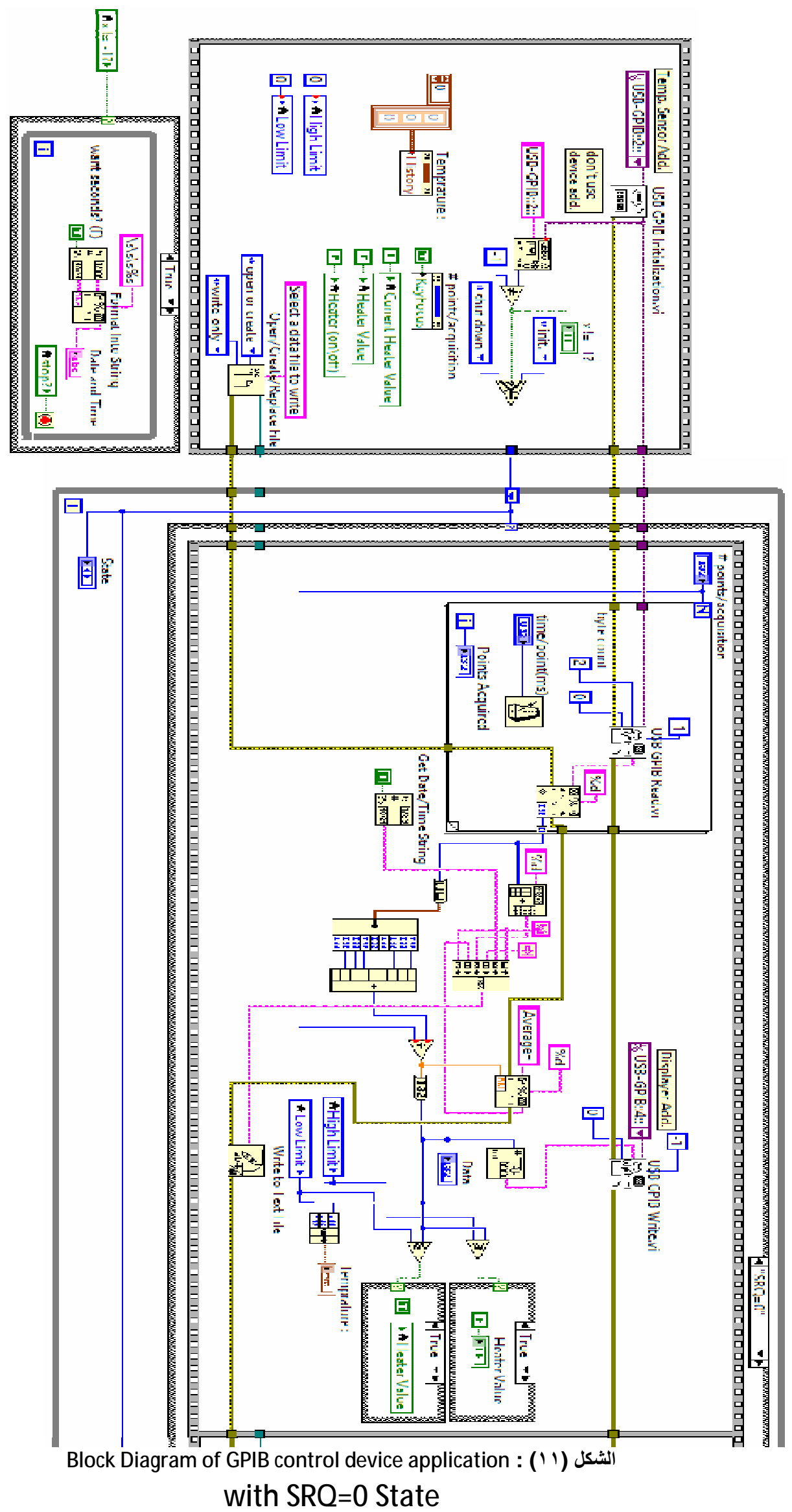




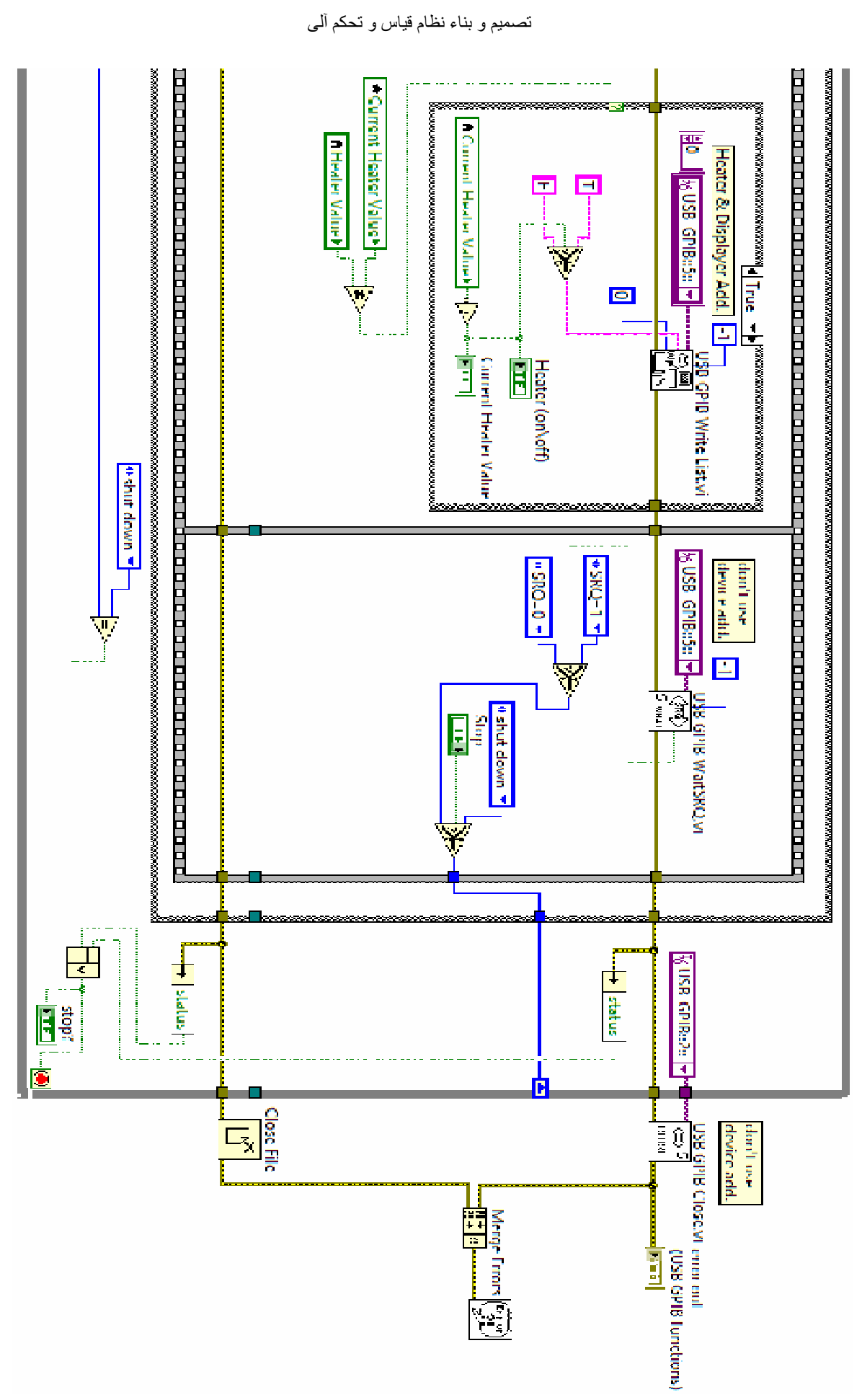

Block Diagram of GPIB control (PC) application : تابع الثكل (r ) with SRQ $=0$ State 


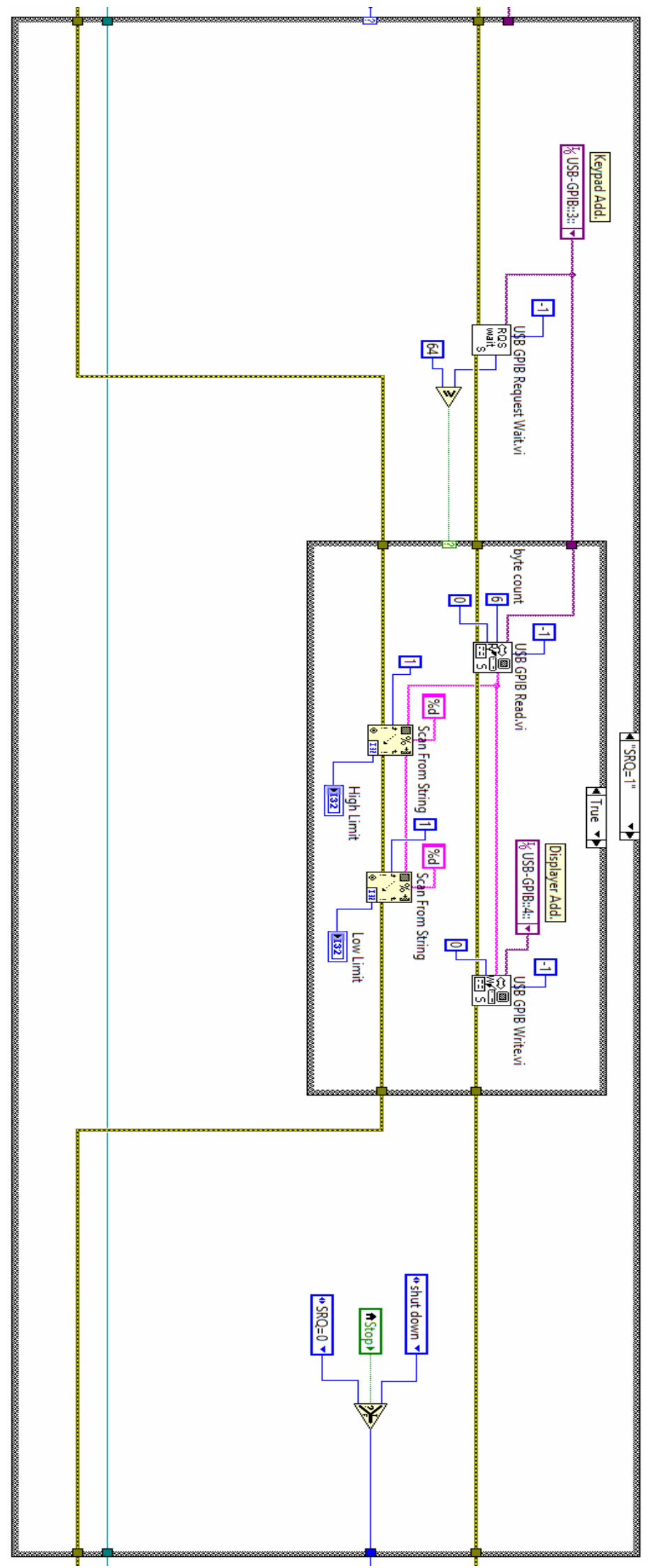

SRQ=1 State of GPIB control (PC) application : الثكل (r I ) 


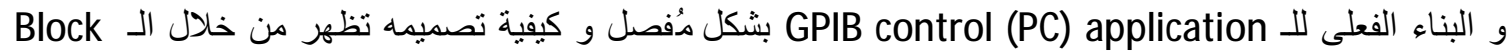

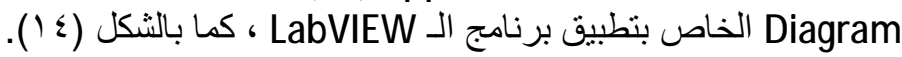

خطوات عمل الـ GPIB Controller Application

خطوات عمل الـ PC application تتم بأربعة مر احل بشكل عام كما فى الثكل (10) ) (كما تم توضيحه فى الـ (Block Diagram و هى كالآتى: (1) (1) (1) (Initialization) init. .( ( )

(main program) SRQ=0.$(r)$

(subroutine) SRQ=1.( $\left({ }^{\top}\right)$ shut down .( $\varepsilon)$

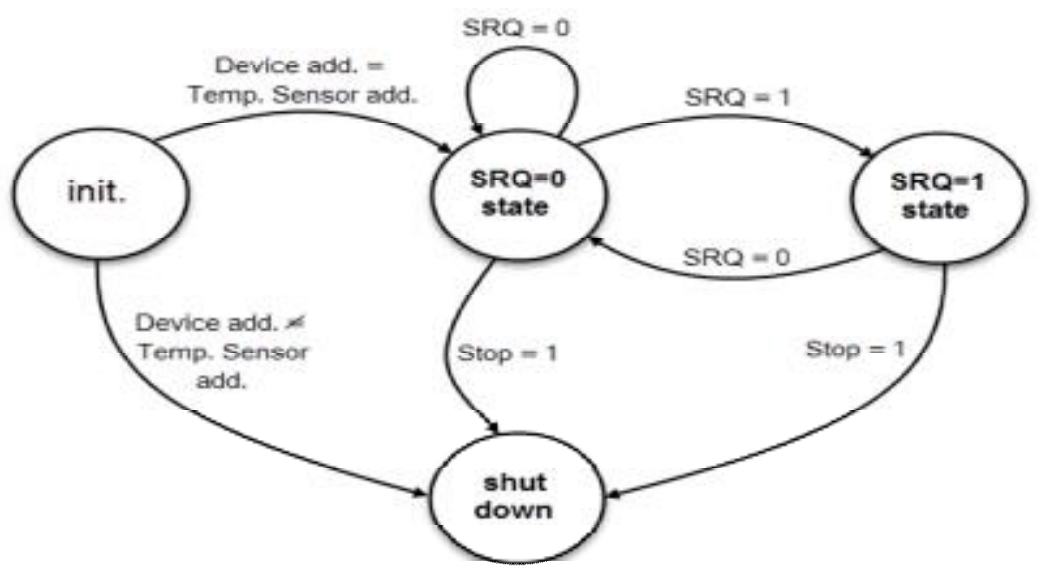

State Machine for software application of PC controller : الثنكل (1 ( )

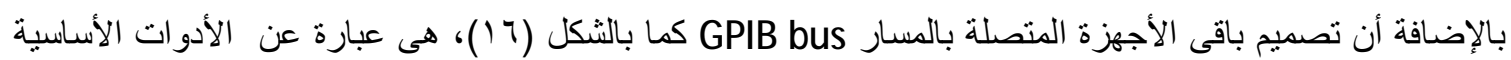

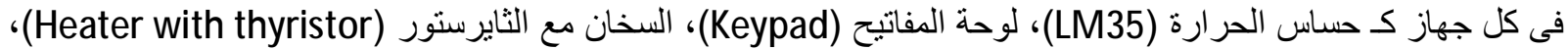

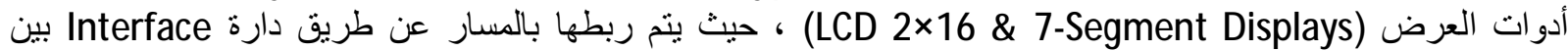
المسار GPIB bus و بين كل أداة من تلك الأدوات. بالإضافة إلى دائرة مصدر طاقة التغذية الذى يمِد عناصر الجه لالار از بالطاقة اللازمة. فالعنصر الأساسى المسؤول عن إدارة كل جهاز هو الدُتحكم الدقيق PIC:16f877a ، حيث تم تصميم كود خاص له له

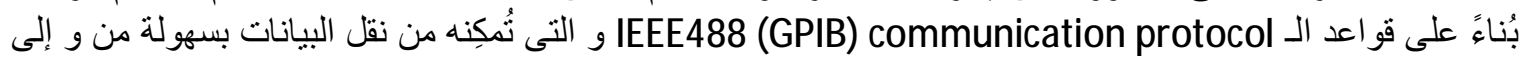
GPIB Bus و الذى بِدوره يسهل تعامل الجهاز بشكل عام مع جهاز التحكم الـ GPIB contloller. 


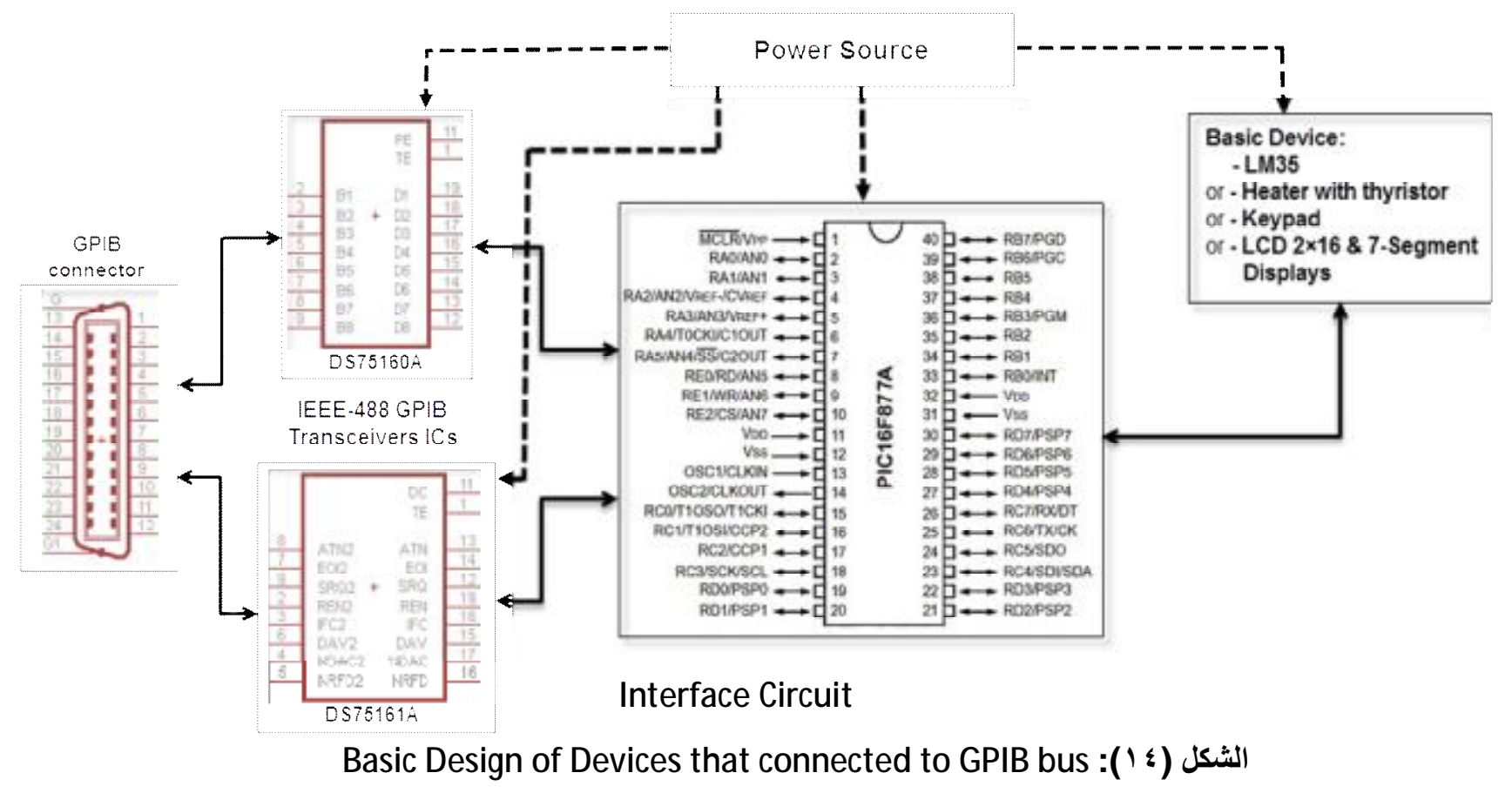

ع ـ-r الطريقة الثالثة(لتنفيذ تصميم و بناء نظم القياس و التحمم الآلى):

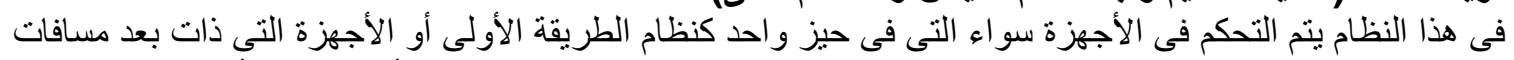

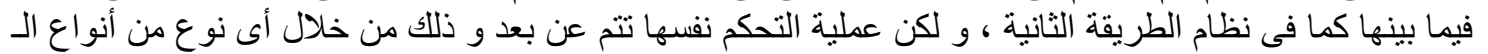
.wireless communication protocols و هنالك طرق و وسائل عديدة لاستخدام برونتوكو لات الاتصال اللاسلكى مع النُّم ، و و خاصةً مع نظام القياس و التحكم

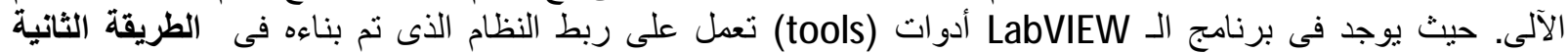

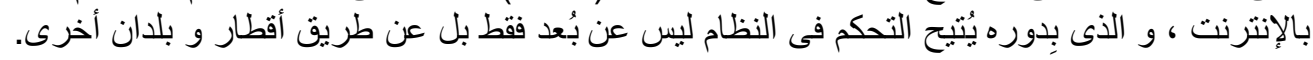

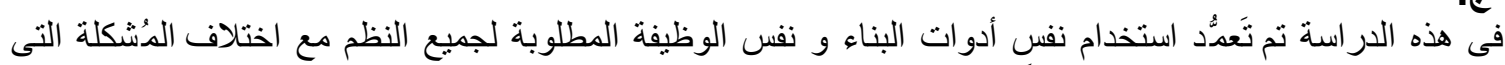

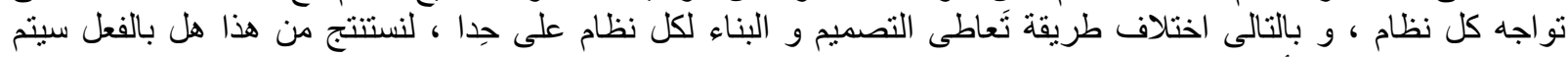

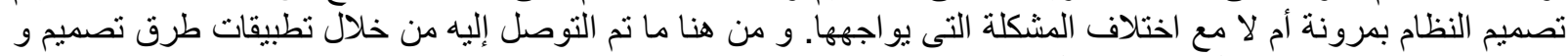

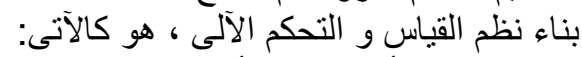

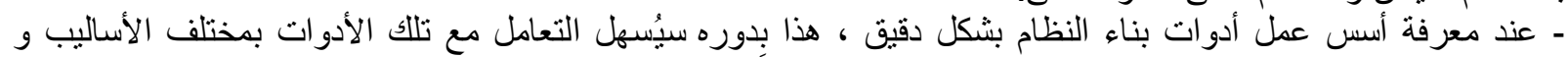

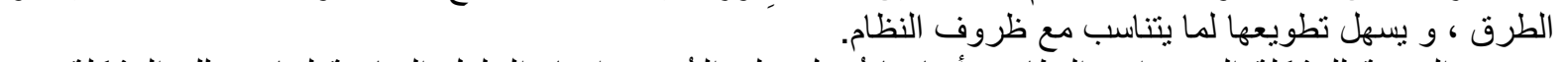

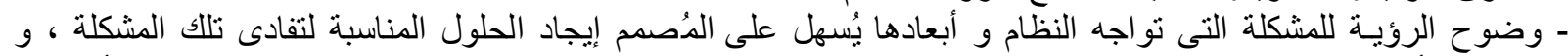

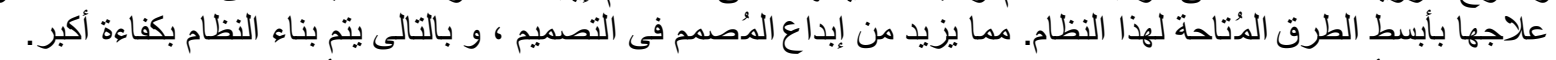

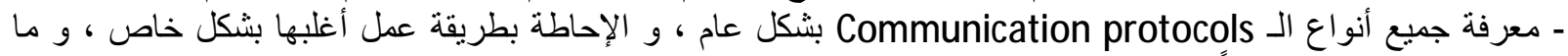

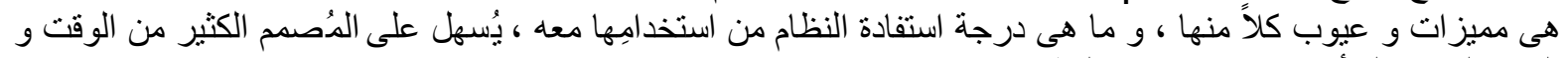
الجهد الذى يبذله أثناء تصميم و بناء النظام نفساء. 


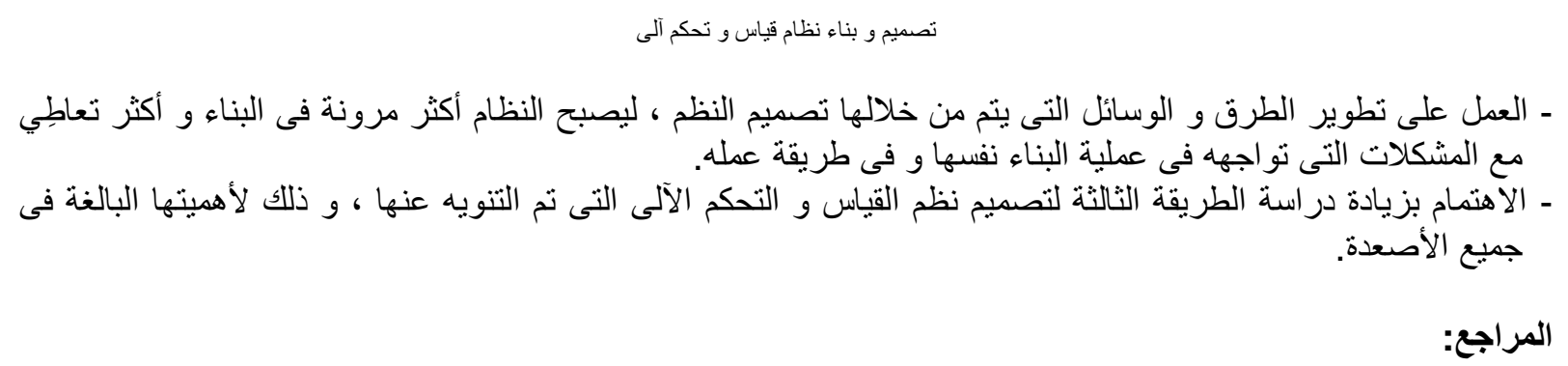

(1). Divya Purohit , Moumita Ghosh, "Challenges and Types of Home Automation Systems", International Journal of Computer Science and Mobile Computing (IJCSMC), Vol. 6, Issue. 4, April 2017, pg.369 - 375.

(2). V. Mazulina, O. Nuyya, E. Sergeeva and N. Vunder, "Digital control system of the recuperation device", 9th Int. Cong. Ultra ModernTelecommunications and Control Systems and Workshops (ICUMT), 6-8 Nov. 2017.

(3). Bogdan Stoyanov, Stefan Stefanov, Jordan Beyazov, Vlaiko Peichev, "Contemporary Methods and Devices for Automatic Measurement", BULGARIAN ACADEMY OF SCIENCES, PROBLEMS OF ENGINEERING CYBERNETICS AND ROBOTICS-57, 2006.

(4). Jim Waldo, " On System Design", Sun Microsystems Lab., Sun Microsystems, USA, 2006.

(5). Craig G. Rieger, David I. Gertman, Miles A. McQueen, " Resilient Control Systems: Next Generation Design Research ", Idaho National Laboratory, Idaho Falls, Idaho, USA, May 2009.

(6). Craig G. Rieger, Kris Villez, " Resilient Control System Execution Agent ", Idaho National Laboratory, Idaho Falls, Idaho, USA, Sept. 2012 IEEE.

(7). Wen-Chiao Lin, Kris R.E. Villez, Humberto E. Garcia, " Experimental validation of a resilient monitoring and control system ", Journal of Process Control, Volume 24, Issue 5, Pages 621-639, May 2014.

(8). YunCui, MyoungjinKim, YiGu, Jong-jinJung, and HankuLee, "Home Appliance Management System for Monitoring Digitized Devices

Using Cloud Computing Technology in Ubiquitous Sensor Network Environment”, Hindawi Publishing Corporation International Journal of

Distributed Sensor Networks Volume 2014, Article ID 174097.

(9). GPIB System Concepts - 492P Programmer's.

(10). B. Glažar, M. Jankovec, M. Topič ,"Development of USB 2.0 Compliant GPIB Controller" , University of Ljubljana, Faculty of Electrical Engineering, Ljubljana, Slovenia, 2005 